\title{
Electric field generation by the electron beam filamentation instability: Filament size effects
}

\author{
M E Dieckmann ${ }^{1}$ and A Bret ${ }^{2}$ \\ 1 VITA, Department of Science and Technology (ITN), Linköping University, \\ Campus Norrköping, 60174 Norrköping, Sweden \\ 2 ETSI Industriales, Universidad de Castilla-La Mancha, 13071 Ciudad Real, Spain \\ E-mail: Mark.E.Dieckmann@itn.liu.se
}

\begin{abstract}
The filamentation instability (FI) of counter-propagating beams of electrons is modelled with a particle-in-cell simulation in one spatial dimension and with a high statistical plasma representation. The simulation direction is orthogonal to the beam velocity vector. Both electron beams have initially equal densities, temperatures and moduli of their nonrelativistic mean velocities. The FI is electromagnetic in this case. A previous study of a small filament demonstrated, that the magnetic pressure gradient force (MPGF) results in a nonlinearly driven electrostatic field. The probably small contribution of the thermal pressure gradient to the force balance implied, that the electrostatic field performed undamped oscillations around a background electric field. Here we consider larger filaments, which reach a stronger electrostatic potential when they saturate. The electron heating is enhanced and electrostatic electron phase space holes form. The competition of several smaller filaments, which grow simultaneously with the large filament, also perturbs the balance between the electrostatic and magnetic fields. The oscillations are damped but the final electric field amplitude is still determined by the MPGF.
\end{abstract}

PACS numbers: 52.35.Qz,52.35.Mw,52.65.Rr 


\section{Introduction}

The filamentation instability (FI) driven by counterpropagating electron beams amplifies magnetic fields in astrophysical and solar flare plasmas [1-5] and it is also relevant for inertial confinement fusion (ICF) [6] and laser-plasma interactions in general [7, 8]. It has been modelled with particle-in-cell (PIC) and Vlasov codes [9-17] taking sometimes into account the ion response and a guiding magnetic field. It turns out that the FI is important, when the beam speeds are at least mildly relativistic and if the beams

have a similar density [18]. Otherwise its linear growth rate decreases below those of the competing two-stream instability or mixed mode instability [19].

The saturation of the FI is attributed to magnetic trapping [9]. More recently, it has been pointed out [13, 14] that the electric fields are also important in this context. An electric field component along the beam velocity vector $\boldsymbol{v}_{b}$ is driven by the FI through the displacement current. This component is typically weak and its relevance to the plasma dynamics is negligible compared to that of the magnetic and the electrostatic fields. The FI is partially electrostatic during its linear growth phase, if the electron beams are asymmetric due to different densities. Symmetric electron beams result in purely electromagnetic waves with wavevectors $\boldsymbol{k} \perp \boldsymbol{v}_{b}$ [19, 20]. A nonlinear growth mechanism is provided in this case by the current of the electrons, which have been accelerated by the magnetic pressure gradient force (MPGF).

The electromagnetic and electrostatic components separate in a $1 \mathrm{D}$ simulation box, because the gradients along two directions vanish in the Maxwell's equations. The electrostatic field is polarized in the simulation direction, while the electromagnetic components are polarized orthogonal to it. If both electron beams have an equal density and temperature, the electrostatic field component along the wavevector $\boldsymbol{k}$ can only be driven nonlinearly. We select here a direction of our 1D PIC simulation box that is orthogonal to $\boldsymbol{v}_{b}$, through which this nonlinear mechanism can be examined in an isolated form. The equally dense and warm counterstreaming beams of electrons have the velocity modulus $\left|\boldsymbol{v}_{b}\right|=0.3 c$. The ions are immobile and compensate the electron charge. The mildly relativistic relative streaming speed $\approx 0.55 \mathrm{c}$ implies, that the growth rate of the FI is significant. At the same time, any relativistic mass changes can be neglected during the growth phase and the saturation of the FI.

The initial conditions of the plasma equal those in the Refs. [21, 22]. The size distribution of the filaments could be sampled with the help of the long 1D simulation box in Ref. [21]. A pair of current filaments, which are small according to this size distribution, has been isolated in Ref. [22]. It could be shown that the electrostatic field is indeed driven by the MPGF for this filament pair. The electrostatic field performed undamped oscillations around a background one. The latter excerted the same force on the electrons as the MPGF. Here we assess the influence of the filament size.

This paper is structured as follows. Section 2 discusses briefly the PIC code, the initial conditions and the key nonlinear processes. The results are presented in the section 3, which can be summarized as follows. The electrons are heated up along the 
wavevector $\boldsymbol{k}$ by their interaction with the wave fields. As we increase the filament size the peak amplitudes grow, which are reached by the magnetic and by the electrostatic

field when the FI saturates. The electron heating increases with the filament size and large electron phase space holes form, which interact with the electromagnetic fields of the filamentation modes. The large box sizes allow the growth of more than one wave and the filamentation modes compete. The electrostatic field oscillations are damped or inhibited and the amplitude modulus converges to one, which equals that expected from the MPGF. We confirm that the strength of the electrostatic force on an electron is comparable to that of the magnetic force, when the FI saturates. The extraordinary modes are pumped by the FI [14]. The results are discussed in section 4.

\section{The PIC simulation, the initial conditions and the nonlinear terms}

The PIC simulation method is detailed in Ref. [23]. Our code is based on the numerical scheme proposed by [24]. The phase space fluid is approximated by an ensemble of computational particles (CPs) with a mass $m_{c p}$ and charge $q_{c p}$ that can differ from those of the represented physical particles. The charge-to-mass ratio must be preserved though. The Maxwell-Lorentz equations are solved. The plasma frequency of each beam with the density $n_{e}$ that we model is $\omega_{p}=\left(e^{2} n_{e} / m_{e} \epsilon_{0}\right)^{0.5}$ and $\Omega_{p}=\sqrt{2} \omega_{p}$. The electric and magnetic fields are normalized to $\boldsymbol{E}_{N}=e \boldsymbol{E} / c m_{e} \Omega_{p}$ and $\boldsymbol{B}_{N}=e \boldsymbol{B} / m_{e} \Omega_{p}$. The current is normalized to $\boldsymbol{J}_{N}=\boldsymbol{J} / 2 n_{e} e c$ and the charge to $\rho_{N}=\rho / 2 n_{e} e$. The physical position, the time and speed are normalized as $x_{N}=x / \lambda_{s}$ with $\lambda_{s}=c / \Omega_{p}, t_{N}=t \Omega_{p}$ and $\boldsymbol{v}_{N}=\boldsymbol{v} / c$. The normalized frequency $\omega_{N}=\omega / \Omega_{p}$. We drop the indices $N$ and $x, t, \omega, \boldsymbol{E}, \boldsymbol{B}, \boldsymbol{J}$ and $\rho$ are specified in normalized units. The equations are

$$
\begin{aligned}
& \nabla \times \boldsymbol{E}=-\partial_{t} \boldsymbol{B}, \nabla \times \boldsymbol{B}=\boldsymbol{J}+\partial_{t} \boldsymbol{E}, \\
& \nabla \cdot \boldsymbol{E}=\rho, \nabla \cdot \boldsymbol{B}=0, \\
& \mathrm{~d}_{t} \boldsymbol{p}_{c p}=q_{c p}\left(\boldsymbol{E}\left[x_{c p}\right]+\boldsymbol{v}_{c p} \times \boldsymbol{B}\left[x_{c p}\right]\right), \mathrm{d}_{x} x_{c p}=v_{c p, x},
\end{aligned}
$$

with $\boldsymbol{p}_{c p}=m_{c p} \Gamma_{c p} \boldsymbol{v}_{c p}$. Here $v_{c p, x}$ is the component along $x$ of $\boldsymbol{v}_{c p}$. The currents $\boldsymbol{j}_{c p} \propto q_{c p} \boldsymbol{v}_{c p}$ of each CP are interpolated to the grid. The summation over all CPs gives $\boldsymbol{J}$, which is defined on the grid. The $\boldsymbol{J}$ updates $\boldsymbol{E}$ and $\boldsymbol{B}$ through (1). Our numerical scheme fulfills (2) as constraints. The new fields are interpolated to the position of each $\mathrm{CP}$ and advance its position $x_{c p}$ and $\boldsymbol{p}_{c p}$ through (3). All components of $\boldsymbol{p}$ are resolved.

Two spatially uniform beams of electrons with $q_{c p} / m_{c p}=-e / m_{e}$ move along $z$. Beam 1 has the mean speed $v_{b 1}=v_{b}$ and the beam 2 has $v_{b 2}=-v_{b 1}$ with $v_{b}=0.3$. Both beams have a Maxwellian velocity distribution in their respective rest frame with a thermal speed $v_{t h}=c^{-1}\left(k_{b} T / m_{e}\right)^{0.5}$ of $v_{b} / v_{t h}=18$. The negative electron charge is compensated by an immobile positive charge background. The initial conditions are $\rho, \boldsymbol{J}, \boldsymbol{E}, \boldsymbol{B}=0$. Figure 1 displays the $\boldsymbol{k}$ spectrum of the unstable waves. The growth rates of the FI modes are close to the maximum value, while relativistic effects are still negligible. The growth rate spectrum with $k_{\|}=0$ relevant for our simulations peaks with $\delta_{M}=0.29$ at $k_{M} \lambda_{s} \approx 10$. A filamentation mode with $k_{M} \lambda_{s}=7$ has been 


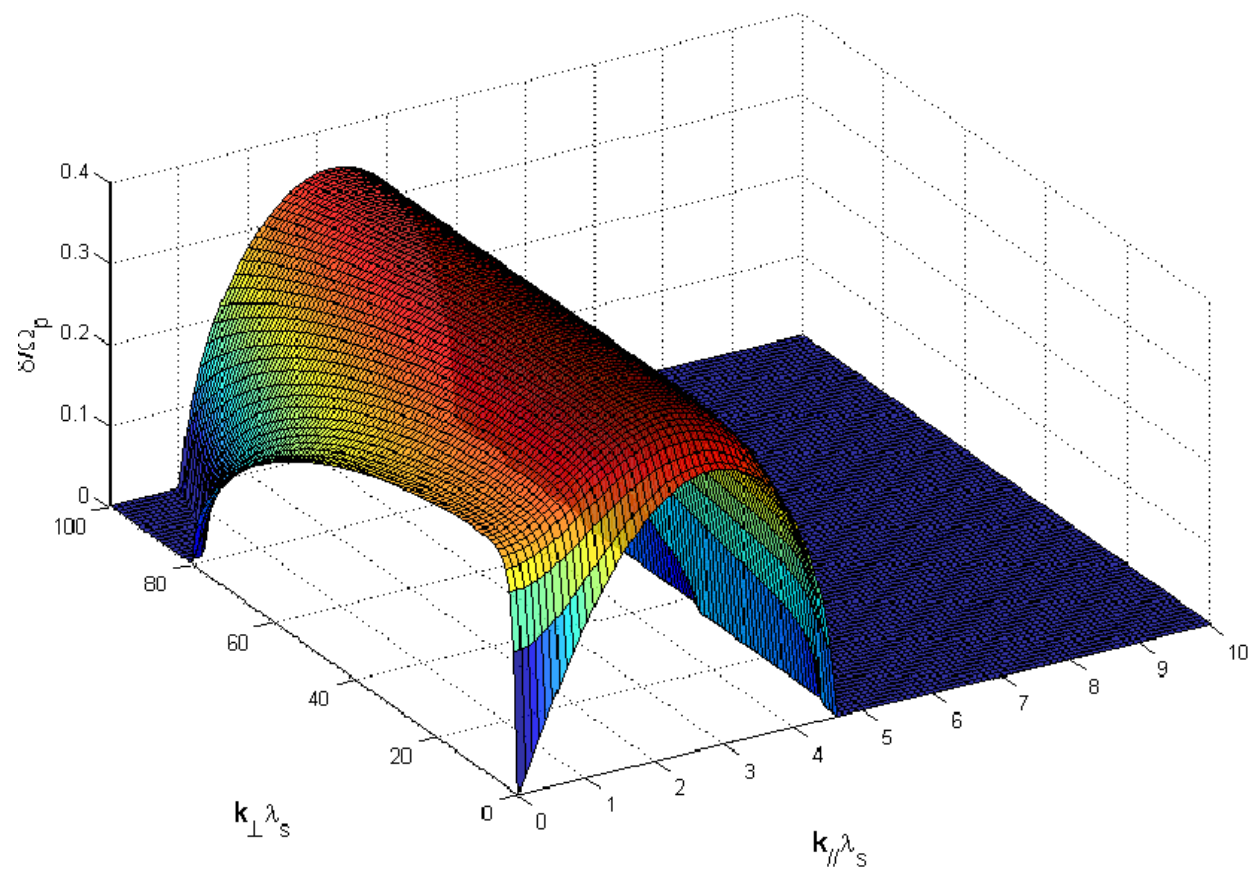

Figure 1. (Colour online) The growth rates in units of $\Omega_{p}$ as a function of the wavenumber in the full $\boldsymbol{k}$ space, where $\lambda_{s} k_{\|}\left(\lambda_{s} k_{\perp}\right)$ points along (orthogonal) to $\boldsymbol{v}_{b}$. The growth rates of the FI modes with $k_{\|}=0$ are comparable to that of the two-stream mode with $k_{\perp}=0$ and to those of the oblique modes. The growth rates for $k_{\|}=0$ decrease to zero for $k_{\perp} \rightarrow 0$ and they are stabilized at high $k_{\perp}$ by thermal effects. The growth rate maximum for $k_{\|}=0$ is $\delta_{M}=0.29$ and it is reached at $k_{M} \lambda_{s} \approx 10$.

considered in detail previously [22], while we investigate here larger filaments. The box length $L_{1}=2$ for the simulation 1 and the filamentation mode with $k_{1}=2 \pi / L_{1}$ grows at the exponential rate $0.92 \delta_{M}$. The box length of the simulation 2 is $L_{2}=2.8$ and the growth rate of the filamentation mode with $k_{2}=2 \pi / L_{2}$ is $0.86 \delta_{M}$. The growth rates decrease rapidly for lower $k$ and these modes are no longer observed in PIC simulations [21]. Both simulations resolve $x$ by $N_{g}=500$ grid cells with the length $\Delta_{x}$ and use periodic boundary conditions. The phase space distributions $f_{1}(x, \boldsymbol{v})$ of beam 1 and $f_{2}(x, \boldsymbol{v})$ of beam 2 are each sampled by $N_{p}=6.05 \cdot 10^{7}$ CPs. The total phase space density is defined as $f(x, \boldsymbol{v})=f_{1}(x, \boldsymbol{v})+f_{2}(x, \boldsymbol{v})$.

Each electron beam constitutes prior to the saturation of the FI a fluid with the index $j$, which has the density $n_{j}(x)=\int_{\boldsymbol{v}} f_{j}(x, \boldsymbol{v}) d \boldsymbol{v}$ and the mean velocity $\boldsymbol{v}_{j}(x)=\int_{\boldsymbol{v}} \boldsymbol{v} f_{j}(x, \boldsymbol{v}) d \boldsymbol{v}$. The normalized momentum equation for such a fluid is

$$
\partial_{t}\left(n_{j} \boldsymbol{v}_{j}\right)+\nabla\left(n_{j} \boldsymbol{v}_{j} \boldsymbol{v}_{j}\right)=-\nabla \mathbf{P}_{j}-n_{j} \boldsymbol{E}+\nabla(\boldsymbol{B} \boldsymbol{B})-\nabla \boldsymbol{B}^{2} / 2+\boldsymbol{B} \times \partial_{t} \boldsymbol{E},(4)
$$

where the thermal pressure tensor $\mathbf{P}_{j}$ is normalized to $2 m_{e} n_{e} c^{2}$. The restriction to one spatial dimension implies, that the gradients along $y$ and $z$ vanish. The FI results in this case in the initial growth of $B_{y}$ and of a weaker electric $E_{z}$. The thermal pressure 
is initially diagonal due to the spatially uniform single-Maxwellian velocity distribution. The $\mathrm{x}$-component of the simplified fluid momentum equation is

$$
\partial_{t}\left(n_{j} v_{j, x}\right)+\mathrm{d}_{x}\left(n_{j} v_{j, x}^{2}\right)=-v_{t h}^{2} \mathrm{~d}_{x} n_{j}-n_{j} E_{x}-B_{y} \mathrm{~d}_{x} B_{y}+B_{y} \partial_{t} E_{z} .
$$

The thermal pressure gradient $v_{t h}^{2} \mathrm{~d}_{x} n_{j}$ is valid, as long as the electron beams have not been heated up. Let us assume that the displacement current and the thermal pressure gradient can be neglected, leaving us with the term $n_{j} E_{x}$ and the MPGF as the key nonlinear terms. The fluid momentum equations can be summed over both beams and we consider the right hand side of (5). As long as $E_{x}$ is small, the electron density is not spatially modulated and $n_{1}+n_{2} \approx 1$. The nonlinear terms cancel out, if $E_{x}=-2 B_{y} \mathrm{~d}_{x} B_{y}$. It could be demonstrated for a short filament in Ref. [22] that this is the case, even when the FI just saturated. The $E_{x}$ oscillated in time and after the saturation with the amplitude $E_{B}=-B_{y} \mathrm{~d}_{x} B_{y}$ around a time-stationary $E_{B}$.

\section{Simulation results}

\subsection{The scaling of $B_{y}, E_{x}$ and $E_{B}$ with the box length}

The beam velocity $\boldsymbol{v}_{b} \| \boldsymbol{z}$ and the electrons of both beams and their micro-currents are re-distributed by the FI only along $x$. The initially charge- and current-neutral plasma is transformed into one with $J_{z}(x, t) \neq 0$. The gradients along the $y, z$-direction vanish in our 1D geometry. Ampere's law simplifies to $d_{x} B_{y}=J_{z}+\partial_{t} E_{z}$, resulting in the growth of $B_{y}$ and $E_{z}$. The MPGF drives $E_{x}$. The $B_{x}=0$ in the $1 \mathrm{D}$ geometry and $E_{y}, B_{z}$ remain at noise levels. The right-hand side of (15) depends on $E_{x}, E_{z}$ and $B_{y}$, as well as on their spatial gradients, which should vary with the filament size.

We want to gain qualitative insight into the scaling of the field amplitudes with the filament size and determine if $E_{x}$ is driven by the MPGF also for the large filaments. The fields that grow in simulation 1 and 2 are compared to those discussed previously in Ref. 22] that used the box size $L_{c}=0.89$. Figure 2 shows the respective dominant Fourier component of $B_{y}$, of $E_{x}$ and of $2 E_{B}$. The amplitude moduli of the mode with $k_{s}=2 \pi / L_{s}$ are considered for $B_{y}$ and those of the $2 k_{s}$ mode for $E_{x}$ and $2 E_{B}$. The subscript $s$ is 1,2 or $c$ and refers to the respective simulation. The amplitudes of $B_{y}$ increase with an increasing box size. After the FI has saturated, we find that $B_{y}\left(k_{1}, t\right) \approx 2 B_{y}\left(k_{c}, t\right)$ and $B_{y}\left(k_{2}, t\right) \approx 2.5 B_{y}\left(k_{c}, t\right)$. The increase of the saturation value of $B_{y}\left(k_{s}, t\right)$ with $L_{s}$ is consistent with magnetic trapping [9]. The magnetic bouncing frequency $\omega_{b}=\left(v_{b} k_{s} B\left[k_{s}, t\right]\right)^{1 / 2}$ in our normalization. The FI should saturate once $\omega_{b}$ is comparable to the linear growth rate of the FI, which is approximately constant for the box sizes $L_{c}, L_{1}$ and $L_{2}$ (Fig. 1). A lower $k_{s}$ supports a larger $B_{y}\left(k_{s}, t\right)$. The $\omega_{b} \approx 0.2$ for simulation 1 is comparable to the linear growth rate $\omega_{i} \approx 0.25$.

After the saturation, the $E_{x}\left(2 k_{1,2}, t\right)>2 E_{x}\left(2 k_{c}, t\right)$ and $E_{x}\left(2 k_{1}, t\right)>E_{x}\left(2 k_{2}, t\right)$. The $E_{x}\left(2 k_{1}, t\right)>3 E_{x}\left(2 k_{c}, t\right)$ while $L_{1} / L_{c} \approx 2.2$. The electrostatic potential in simulation 1 is thus larger by a factor 6 , which should result in a more violent electron acceleration than in the box with the length $L_{c}$. The thermal pressure gradient force is potentially 

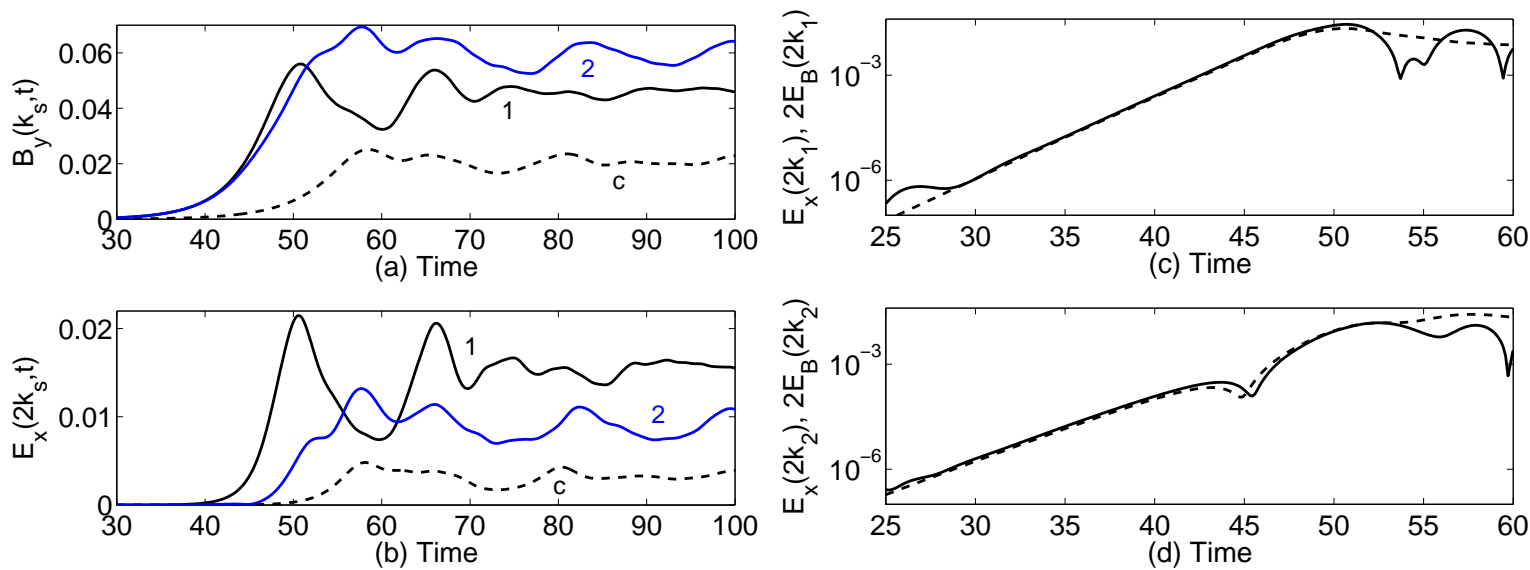

Figure 2. (Colour online) Panel (a) compares the $B_{y}\left(k_{s}, t\right)$ and panel (b) the $E_{x}\left(2 k_{s}, t\right)$ in the boxes with the size $L_{1}, L_{2}$ and $L_{c}$ (dashed curve). $E_{B}\left(2 k_{s}, t\right)$ (dashed curve) is compared with $E_{x}\left(2 k_{s}, t\right)$ (solid curves) for the box size $L_{1}(\mathrm{c})$ and $L_{2}$ (d).

more important for larger filaments and it may modify the balance between the nonlinearly driven $E_{x}$ and the MPGF. However, an excellent match between $E_{x}\left(2 k_{1}, t\right)$ and $2 E_{B}\left(2 k_{1}, t\right)$ is observed for $t<50$, due to which the two nonlinear terms on the right hand side of (5) practically cancel for simulation 1 . The $E_{x}\left(2 k_{2}, t\right) \approx 2 E_{B}\left(2 k_{2}, t\right)$ in simulation 2 for $30<t<42$ and for $46<t<53$. Both fields disagree in between these time intervals and a local minimum is observed. The field and electron dynamics is now examined in more detail for the box lengths $L_{1}$ and $L_{2}$.

\subsection{Simulation 1: Box length $L_{1}=2$}

Figure 3 displays the evolution of the relevant field components. The $B_{y}(x, t)$ rapidly grows and saturates at $t \approx 45$. It is initially stationary in space but it oscillates in time until $t \approx 65$, which implies that $B_{y}(x, t)$ does not immediately go into its stable saturated state. The $B_{y}(x, t)$ shows only one spatial oscillation and the filamentation mode with the wavelength $k_{1}=2 \pi / L_{1}$ is thus strongest. However, the interval with the large positive $B_{y}(x, t \approx 45)$ covers $0<x<0.9$, while that with the large negative $B_{y}(x, t \approx 45)$ is limited to $1.2<x<1.7$. This mode is thus initially not monochromatic. The saturated structure formed by $B_{y}(x, t)$ drifts after $t \approx 65$ to lower $x$ at a speed $<0.01$ and it remains stationary in its moving rest frame. The $E_{z}(x, t)$ grows initially in unison with $B_{y}(x, t)$ and it is shifted in space by $90^{\circ}$ with respect to $B_{y}(x, t)$, as expected from Ampere's law. Oscillations of $E_{z}(x, t)$ are spatially correlated with those of the $B_{y}(x, t)$ for $45<t<65$. The $E_{z}(x, t)$ undergoes a mode conversion at $t \approx 65$ into a time-oscillatory and spatially uniform $E_{z}(x, t)$. Figure 3(c) demonstrates that $E_{x}(x, t)$ is following the drift of $B_{y}(x, t)$ towards decreasing $x$, but that its wavenumber is twice that of $B_{y}(x, t)$. The $B_{y}(x, t)$ is stationary in its moving rest frame, while $E_{x}(x, t>70)$ is oscillating around an equilibrium electric field with an amplitude and spatial distribution that resembles $E_{B}(x, t)$ in Fig. 3(d). The electric and the magnetic 


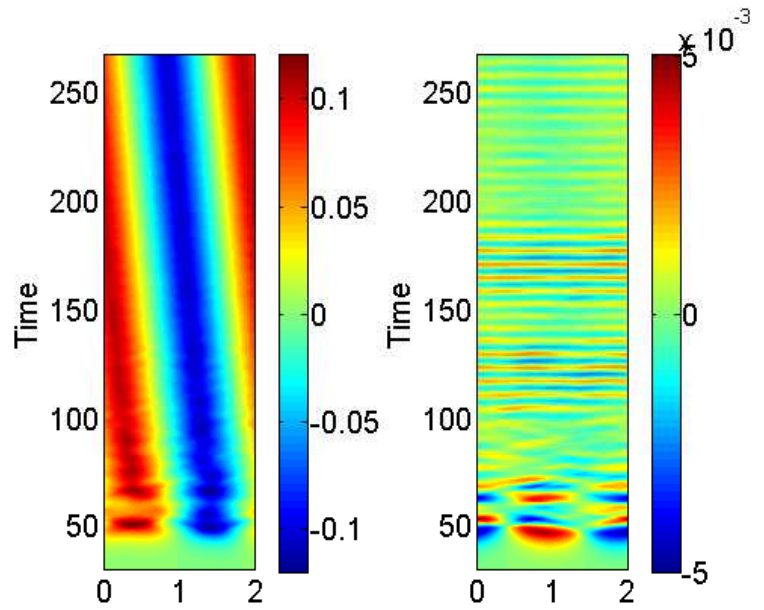

(a) Position

(b) Position

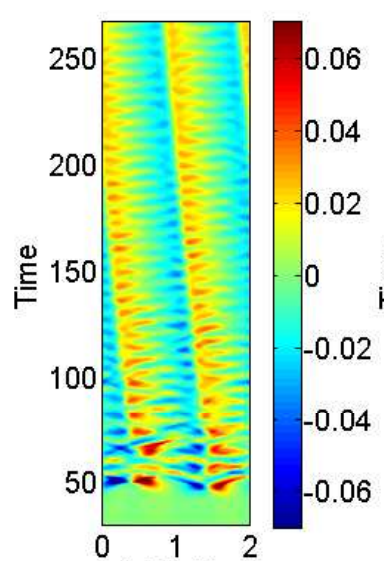

(c) Position

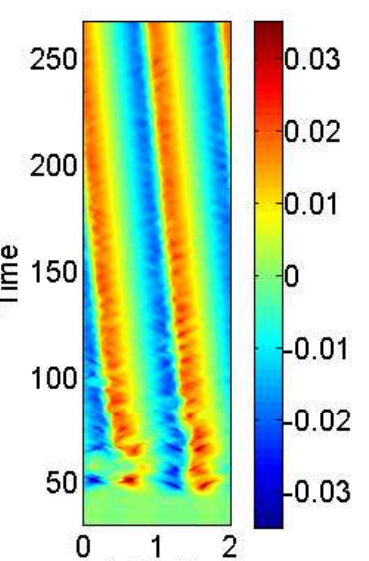

(d) Position

Figure 3. (Colour online) The field amplitudes in the box $L_{1}$ : The panels (a-d) show $B_{y}, E_{z}, E_{x}$ and $E_{B}$, respectively. The amplitude of $B_{y}$ reaches a time-stationary distribution, which convects to decreasing $x$ at a speed $<0.01$. The $E_{z}$ and $E_{x}$ components are oscillatory in space and in time. The $E_{z}$ is phase-shifted by $90^{\circ}$ relative to $B_{y}$ when the fields saturate at $t \approx 45$. The $E_{x}$ and the $E_{B}$ are co-moving and $E_{x}$ oscillates in time around a mean amplitude comparable to $E_{B}$ for $t>70$.

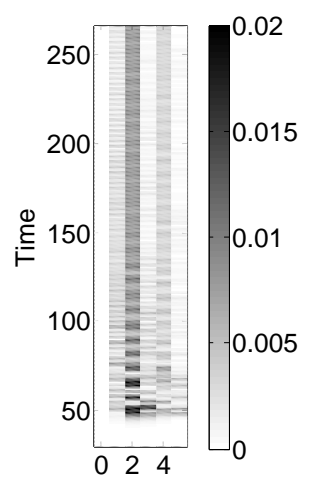

(a) $\mathrm{k} / \mathrm{k} 1$

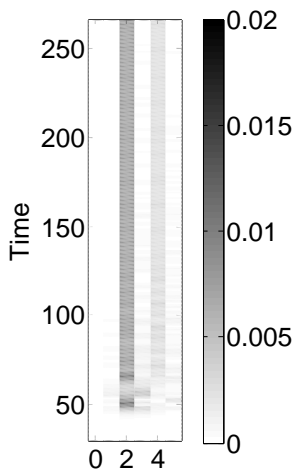

(b) $k / k 1$
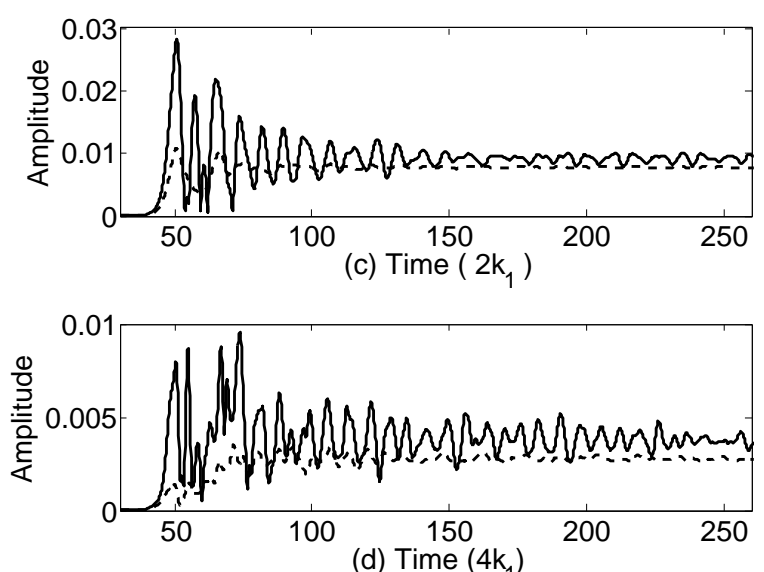

Figure 4. The relevant part of the amplitude spectrum $E_{x}(k, t)$ is displayed for low $k$ in (a) and (b) shows that of $E_{B}(k, t)$. The wavenumbers are expressed in units of $k_{1}$. The amplitude moduli of the dominant modes are displayed for $k=2 k_{1}$ in (c) and its first harmonic with $k=4 k_{1}$ in (d), where the dashed curves correspond to $E_{B}$.

forces are comparable in their strength, but their distribution differs.

Figure 4 compares in more detail the moduli of the amplitude spectra $E_{x}(k, t)$ and $E_{B}(k, t)$. The amplitudes of the strongest modes fulfill $E_{x}\left(2 k_{1}, t\right) \approx 2 E_{B}\left(2 k_{1}, t\right)$ until $t=50$ (See also Fig. 2). The $E_{x}\left(2 k_{1}, t\right)$ thus overshoots $E_{B}\left(2 k_{1}, t\right)$ and it oscillates around it after $t=50$. The oscillation is damped and the amplitudes of $E_{x}\left(2 k_{1}, t\right)$ and $E_{B}\left(2 k_{1}, t\right)$ converge. The full spectra $E_{x}(k, t)$ and $E_{B}(k, t)$ reveal that the mode $k=4 k_{1}$ is also important for $t>100$. It probably is a harmonic of the mode with $k=2 k_{1}$ and 

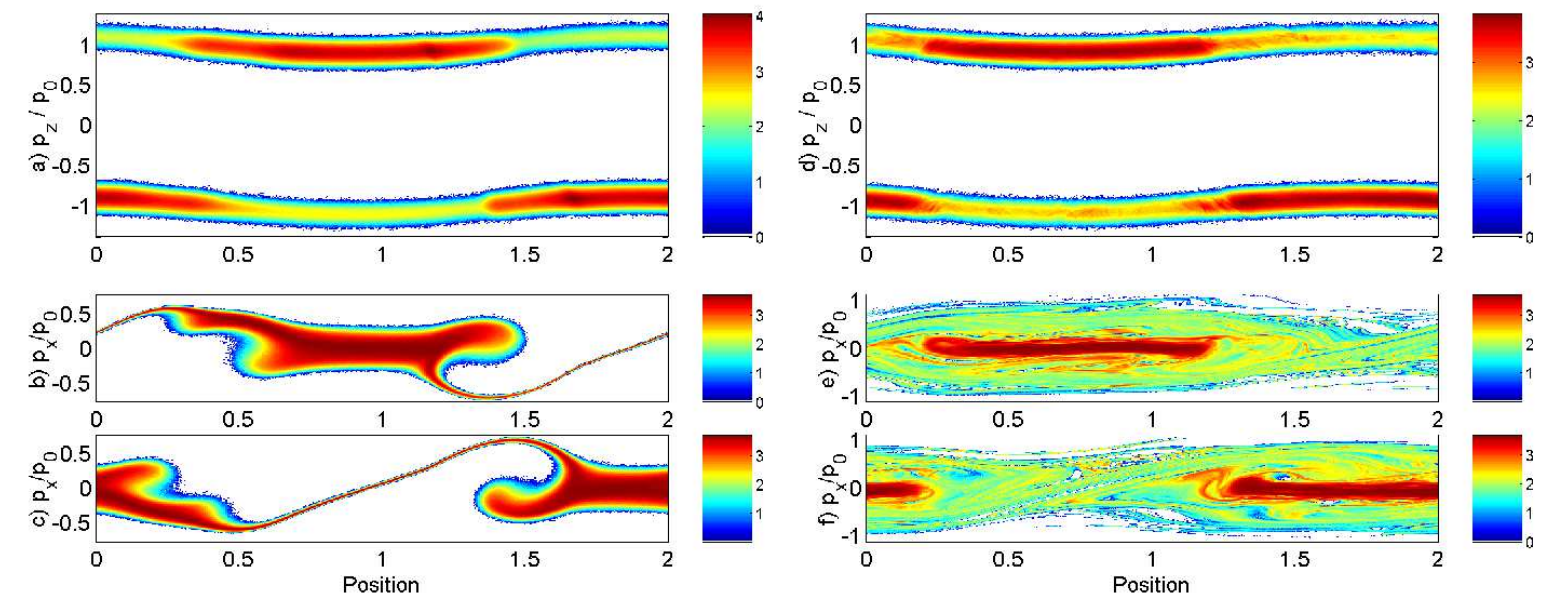

Figure 5. (Colour online) The 10-logarithm of the phase space densities in units of CPs at the time $t=50(\mathrm{a}-\mathrm{c})$ and $t=120(\mathrm{~d}-\mathrm{f})$ in the box $L_{1}$ : Panels (a,d) show the total phase space density $f\left(x, p_{z}\right)$ with the beam momentum $p_{0}=m_{e} v_{b} \Gamma\left(v_{b}\right)$. The phase space density $f_{1}\left(x, p_{x}\right)$ of beam 1 is shown in (b,e) and the $f_{2}\left(x, p_{x}\right)$ of beam 2 in $(\mathrm{c}, \mathrm{f})$. Both beams are spatially separated and $(\mathrm{e}, \mathrm{f})$ reveal cool electron clouds immersed in a hot electron background with momenta of up to $\approx p_{0}$.

not an independently growing FI mode. Otherwise we would expect that the mode with $k \approx 3 k_{1}$ also grows. The amplitude of $E_{x}\left(4 k_{1}, t\right)$ is close to that of $E_{B}\left(4 k_{1}, t\right)$.

A dissipation mechanism for the interplaying $J_{x}$ and $E_{x}$ is present, which causes the damping and the convergence of $E_{x}(x, t)$ to $E_{B}(x, t)$. The damping persists after $t=65$, when $B_{y}$ is quasi-stationary in its moving reference frame. The term $B_{y} \partial_{t} E_{z}$ in (5) could, in principle, be one dissipation mechanism. However, even at $t \approx 50$ when $\partial_{t} E_{z}$ is largest and $B_{y}$ has developed in full, this term is weaker by more than one order of magnitude than the MPGF and the term $n_{j} E_{x}$ in simulation 1 (not shown). If the term $B_{y} \partial E_{z}$ would be the damping mechanism, this should have resulted in a noticable field damping also in the short simulation box with length $L_{c}$. A damping of $E_{x}(x, t)$ has not been observed in Ref. [22]. The thermal pressure gradient force may provide this damping and we examine now the electron phase space density distribution.

Figure 5 displays the phase space distributions $f_{1}\left(x, p_{x}\right)$ of the beam 1 and the $f_{2}\left(x, p_{x}\right)$ of the beam 2 at the times $t=50$ and $t=120$. The total phase space density $f\left(x, p_{z}\right)$ is shown for the same times. The beams reveal a high degree of symmetry already at $t=50$ and the filament centres are shifted along $x$ by $L_{1} / 2$. The phase space structures in Fig. 5 (b,c) are, however, different at the filament boundaries $x \approx 0.5$ and $x \approx 1.5$. This difference is responsible for the deviation of the initial $B_{y}(x, t)$ from a sine curve in Fig. 3(a). The phase space distribution at late times reveals, that the electrons are heated along $p_{x}$ but not along $p_{z}$. The filament drift to lower $x$ is visible from Figs. 5(a,d) and agrees with the observed one of $B_{y}(x, t)$ in Fig. 3 , The electrons are accelerated along $x$ to a peak speed $\sim v_{b}$, which is more than twice that observed in the box with the length $L_{c}[22$. The peak electron kinetic energy due to the velocity 


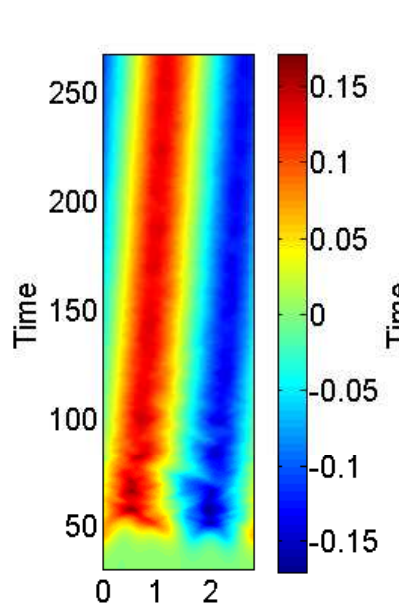

(a) Position

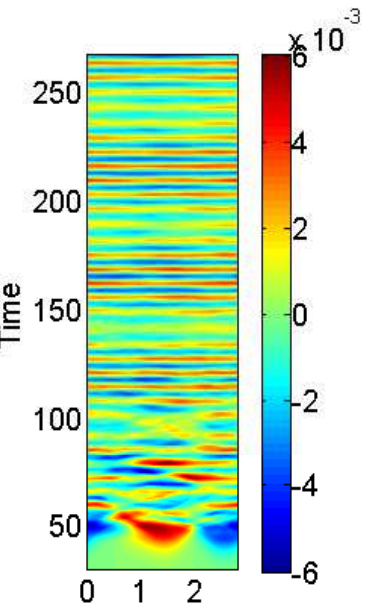

(b) Position

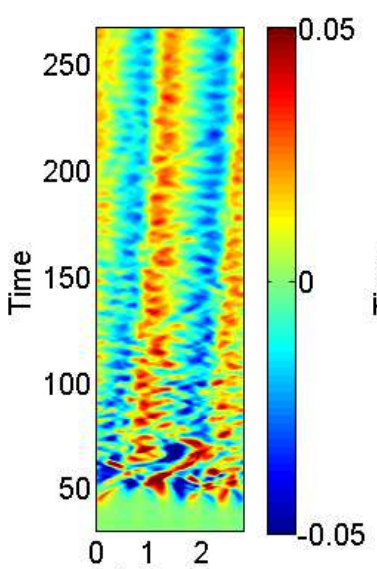

(c) Position

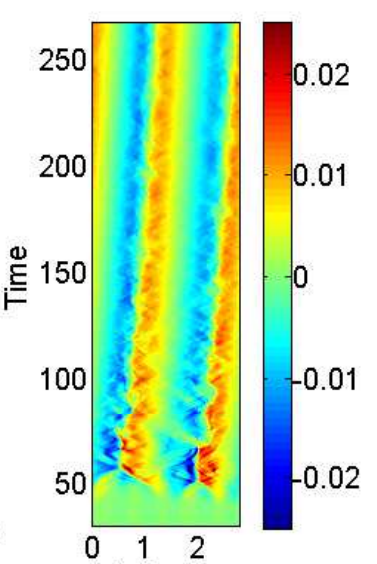

(d) Position

Figure 6. The field amplitudes in the box $L_{2}$ : The panels (a-d) show $B_{y}, E_{z}, E_{x}$ and $E_{B}=-B_{y} d_{x} B_{y}$, respectively. The amplitude of $B_{y}$ reaches a steady state value, which convects to increasing $x$ at a speed $<0.01$. The $E_{z}$ and $E_{x}$ components are oscillatory in space and in time. The $E_{z}$ is phase-shifted by $90^{\circ}$ relative to $B_{y}$ when the fields saturate at $t \approx 50$. The $E_{x}$ and the $E_{B}$ are co-moving and $E_{x}(x)$ oscillates in time around a mean amplitude comparable to $E_{B}$ for $t>100$.

component along $x$ thus increases by a factor, which is comparable to the increase in the electrostatic potential as we go from a box with length $L_{c}$ to one with $L_{1}$. This strong electron heating is likely to result in higher thermal pressure gradient forces. The expression $\mathrm{d}_{x} n_{1}(x) \int v_{x} f\left(x, v_{x}\right) d v_{x}$ has been evaluated (not shown) at $t=75$ and its peaks reach values $\approx 0.1$, which are comparable to the MPGF. The width of these peaks is small compared to the electron skin depth.

Movie 1 animates in time the 10-logarithmic phase space distributions $f_{1}\left(x, p_{x}\right)$ and $f_{1}\left(x, p_{z}\right)$ of the beam 1 in the simulation 1 . The formation of the filaments is demonstrated. We observe a dense untrapped electron component immersed in an electron cloud that has been heated along the simulation direction by the saturation of the FI. The spatial width of the plasmon containing the dense bulk of the confined electrons in $f_{1}\left(x, p_{x}\right)$ oscillates in time. The overlap of the filaments in Fig. $5(\mathrm{e}, \mathrm{f})$ is thus time dependent and related through its current $J_{x}(x, t)$ to the oscillating $E_{x}(x, t)$ in Fig. 3(b). The phase space distribution $f_{1}\left(x, p_{x}\right)$ reveals small-scale structures (phase space holes) that gyrate around the centre of the filament. These coherent structures result in jumps in the thermal pressure.

\subsection{Simulation 2: Box length $L_{2}=2.8$}

Figure 6 displays the fields that grow in the simulation with the box length $L_{2}=2.8$. The growth rate map in Fig. 1 demonstrates that the FI can drive simultaneously several modes in the simulation box. The mode with $k_{2}=2 \pi / L_{2} \approx 2.25$ has, for example, a lower growth rate than that with $k \approx 2 k_{2}$. We observe consequently oscillations 


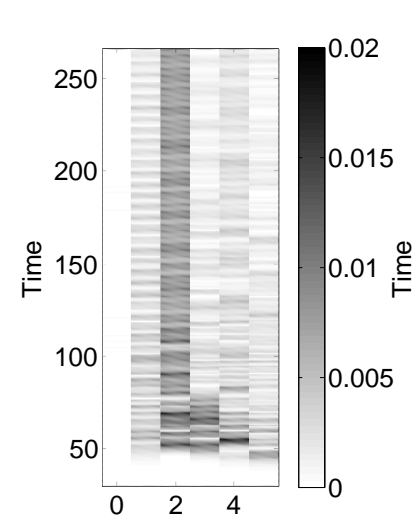

(a) $k / k 2$
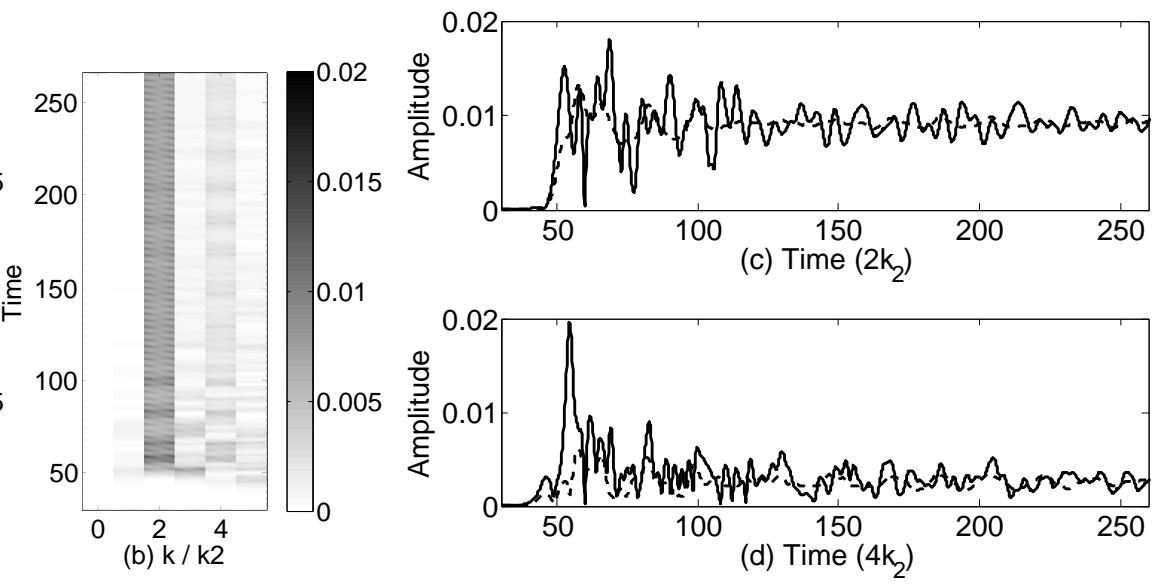

Figure 7. The relevant part of the amplitude spectrum $E_{x}(k, t)$ is displayed for low $k$ in (a) and (b) shows that of $E_{B}(k, t)$. The wavenumbers are expressed in units of $k_{2}$. The amplitude moduli of the dominant modes are displayed for $k=2 k_{2}$ in (c) and its first harmonic with $k=4 k_{2}$ in (d), where the dashed curves correspond to $E_{B}$.

in $B_{y}(x, t)$ along $x$, which are a superposition of several waves with a $k \geq k_{2}$ during the initial growth phase $40<t<50$. These oscillations merge and only one spatial oscillation of $B_{y}(x, t)$ and, thus, a single pair of filaments survive after the saturation at $t \approx 50$. The magnetic field structure convects to increasing values of $x$ at a speed $<0.01$, but it is stationary in its rest frame after $t \approx 70$. The phase of $E_{z}(x, t)$ is shifted by $90^{\circ}$ with respect to $B_{y}(x, t)$ for $40<t<60$. The oscillations of $E_{z}(x, t)$ undergo a mode conversion during $60<t<100$ and we observe undamped oscillations with $k=0$ for $t>100$. The amplitude of these oscillations is modulated on a long timescale. The $E_{x}(x, t)$ and the $B_{y}(x, t)$ show no correlation until $t \approx 70$. Thereafter the spatial amplitude of $E_{x}(x, t)$ oscillates in time around $E_{B}(x, t)$. The force on an electron imposed by $E_{x}(x, t)$ is comparable to that imposed by $v_{b} B_{y}(x, t)$.

A more accurate comparison of $E_{x}(x, t)$ and $E_{B}(x, t)$ is again provided by the moduli of their spatial amplitude (Fourier) spectra, $E_{x}(k, t)$ and $E_{B}(k, t)$. Figure 7 displays $E_{x}(k, t)$ and $E_{B}(k, t)$ and compares in more detail $E_{x}\left(2 k_{2}, t\right)$ with $E_{B}\left(2 k_{2}, t\right)$ as well as $E_{x}\left(4 k_{2}, t\right)$ with $E_{B}\left(4 k_{2}, t\right)$. The amplitudes $E_{x}\left(2 k_{2}, t\right) \approx 2 E_{B}\left(2 k_{2}, t\right)$ during the exponential growth phase of the FI for $25<t<45$ (See Fig. 2), the amplitude moduli then have a local minimum and continue to grow after this time. We identify the likely reason from $E_{B}(k, t)$ in Fig. $7(\mathrm{~b})$. The $E_{B}\left(3 k_{2}, t\right)$ competes with $E_{B}\left(2 k_{2}, t\right)$ at $t \approx 50$.

A large amplitude modulus of $E_{B}\left(3 k_{2}, t\right)$ evidences that $B_{y}(x, t)$ is not a sine wave at this time. If $B_{y} \propto \sin \left(k_{2} x\right)$, then $E_{B} \propto \sin \left(k_{2} x\right) \cos \left(k_{2} x\right)$ and $E_{B}(k, t)$ would be composed of a wave with $k=2 k_{2}$. The periodic boundary conditions would also allow for a $B_{y} \propto \sin \left(2 k_{2}, t\right)$ and here $E_{B}$ would involve a wave with $k=4 k_{2}$. An $E_{B}\left(3 k_{2}, t\right)$ can thus not be connected to a single filamentation mode. During the linear growth phase of the FI prior to $t \approx 40$, the $J_{z}(x, t)$ can form structures with a wideband wavenumber spectrum (See Fig. 1) and their associated $B_{y}$ can grow independently. 

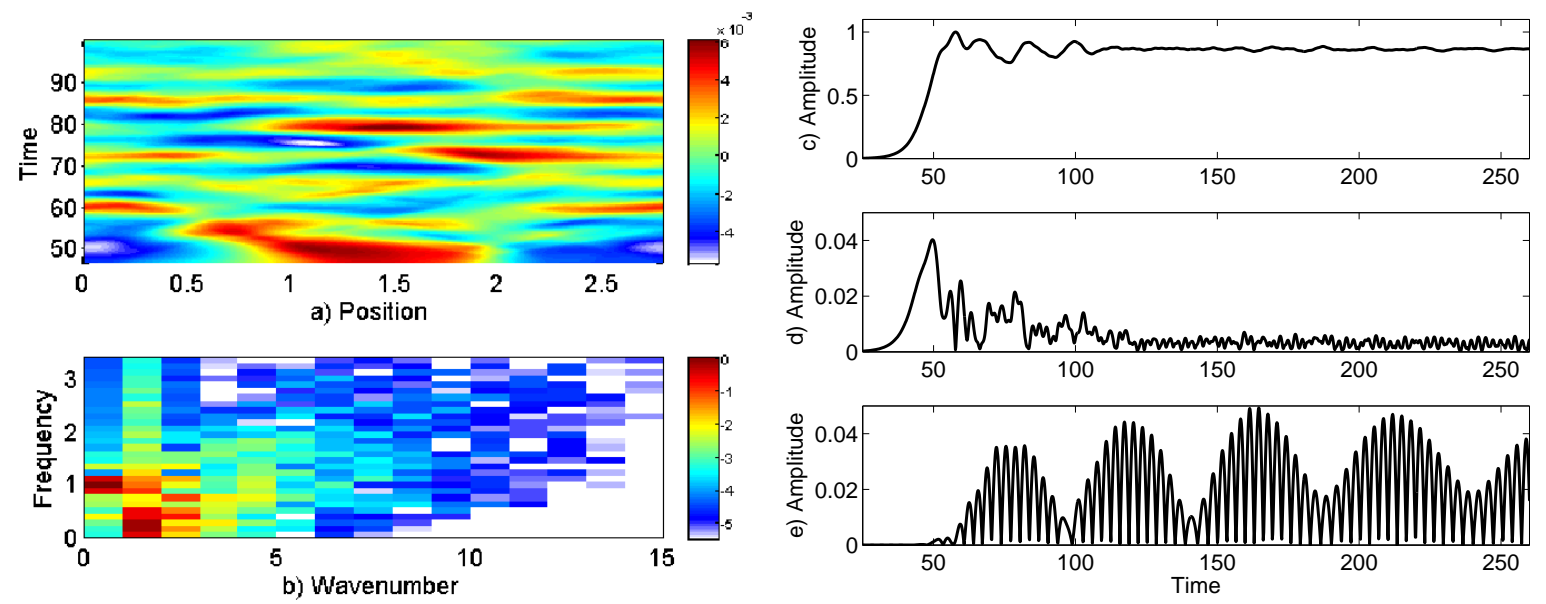

Figure 8. (Colour online) A time-interval of $E_{z}(x, t)$ and the 10-logarithm of its power spectrum $P_{E Z}(k, \omega)$ are displayed in (a) and (b). Wavenumbers are given in units of $k_{2}$. Peak 1 is at $\omega<0.5$ and $k=k_{2}$. Peak 2 is observed at $k=k_{2}$ and $\omega \approx 1$ and peak 3 at $k=0$ and $\omega \approx 1$. The $B_{y}\left(k_{2}, t\right)$ is shown in $(\mathrm{c})$, the $E_{z}\left(k_{2}, t\right)$ in $(\mathrm{d})$ and $E_{z}(0, t)$ in (e), all normalized to the maximum of $B_{y}\left(k_{2}, t\right)$.

Once the MPGF in Eq. 5 has reached a significant strength, the FI saturates. The strength of the MPGF increases with $k$, due to the larger $\mathrm{d}_{x} B_{y}(x, t)$ of the rapid oscillations. The $B_{y} \propto \sin \left(k_{2} x\right)$ should maximize the magnetic field strength for a given MPGF. This may explain why this mode is the dominant one after $t=70$ despite its lower growth rate. The decrease of $E_{B}\left(2 k_{2}, t\right)$ in Fig. $2(\mathrm{~d})$ at $t \approx 45$ is tied to the saturation of $E_{B}\left(3 k_{2}, t\right)$. The $E_{x}(k, t)$ in Fig. 7(a) has a broadband spectrum within $50<t<75$, which is probably caused by the current $J_{x}$ arising from the rearrangement of the filaments. After this time, $E_{x}\left(2 k_{2}, t\right) \approx E_{B}\left(2 k_{2}, t\right)$ and $E_{x}\left(4 k_{2}, t\right) \approx E_{B}\left(4 k_{2}, t\right)$. The $E_{x}\left(2 k_{2}, t\right)$ does not show oscillations around $E_{B}\left(2 k_{2}, t\right)$ as the simulation 1 . The filament rearrangement inhibits an oscillatory equilibrium between $J_{x}$ and $E_{x}$.

Figure 8 examines the mode conversion of the electromagnetic $E_{z}$ component observed in Fig. 6(b). The $P_{E Z}(k, \omega)$ is the squared modulus of the Fourier transform of $E_{z}(x, t)$ over space and over $45<t<100$. The dispersion relation shows three peaks. Peak 1 has a $k=k_{2}$ and $\omega<0.5$ and it is tied to the $E_{z}(x, t)$ of the FI mode. This mode grows exponentially and aperiodically. Its frequency spectrum is thus spread out along $\omega$. Its energy can leak into the peak 2 at $k=k_{2}$ and $\omega \approx 1$. The $E_{z}(x, t)$ is orthogonal to $B_{y}(x, t)$ and peak 2 corresponds to an extraordinary mode, similar to the slow extraordinary mode. Peak 3 has a $k=0$ and $\omega \approx 1$ and it corresponds to a spatially uniform oscillation in an extraordinary mode branch. The intermittent behaviour of $E_{z}(x, t)$ in Fig. 8(a) results in a broadband spectrum in $k$ and $\omega$. These turbulent wave fields can couple energy directly to the high-frequency electromagnetic modes and excite a discrete spectrum if the boundary conditions are periodic [14].

The interplay of the waves belonging to the three peaks in Fig. 8(a) is assessed with the moduli of the amplitude spectra $B_{y}\left(k_{2}, t\right), E_{z}\left(k_{2}, t\right)$ and $E_{z}(0, t)$ in Figs. 8)(c- 

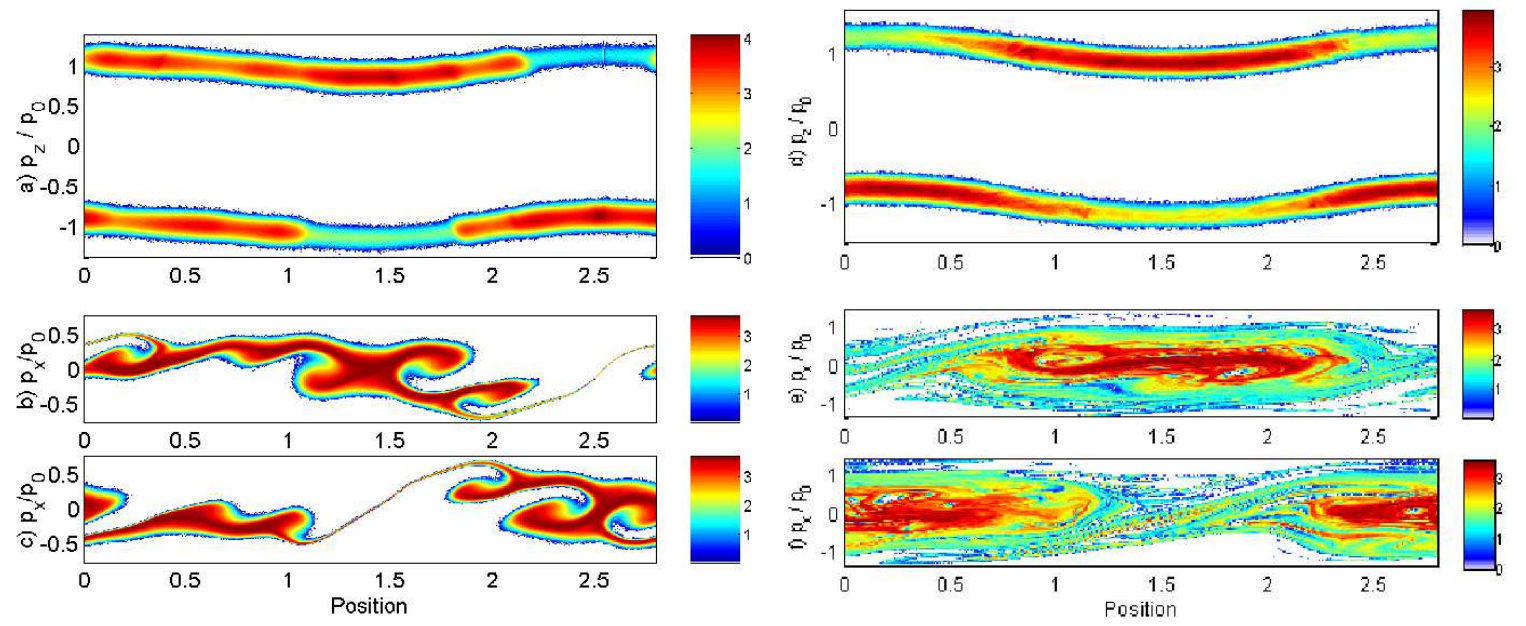

Figure 9. The 10-logarithmic phase space densities in units of CPs at $t=50$ (a-c) and $t=120(\mathrm{~d}-\mathrm{f})$ in the box $L_{2}$ : Panels (a,d) show the total distribution $f\left(x, p_{z}\right)$ with $p_{0}=m_{e} v_{b} \Gamma\left(v_{b}\right)$. The beam temperature along $p_{z}$ is unchanged. The distribution $f_{1}\left(x, p_{x}\right)$ of beam 1 is shown in (b,e) and the $f_{2}\left(x, p_{x}\right)$ of beam 2 in $(\mathrm{c}, \mathrm{f})$. The electrons of both beams spatially separate and (e,f) reveal a dense electron component immersed in a tenuous hot electron background, which reaches a thermal width $\approx p_{0}$.

e). The $B_{y}\left(k_{2}, t\right)$ and $E_{z}\left(k_{2}, t\right)$ grow at the same exponential rate until they saturate at $t \approx 50$, evidencing that they belong to the same FI mode. The $B_{y}\left(k_{2}, t\right)$ maintains its amplitude after $t=50$, while $E_{z}\left(k_{2}, t\right)$ decreases until $t \approx 120$ and remains constant thereafter. The $E_{z}(0, t)$ grows in the same time interval to its peak amplitude, which suggests a parametric interaction between these modes. The amplitude modulation in Fig. 8(e) must be caused by a beat between two waves, which are similar to the slow- and fast extraordinary modes in the limit $k=0$. Both modes are undamped on the resolved timescales. One may interpret the parametric interaction as a three-wave coupling between the waves corresponding to the peaks 1-3 in Fig. 8(b), resembling the system of Ref. [25]. However, here the $B_{y}(x, t)$ varies spatially and the parametric interaction may involve more of the waves of the spectrum in Fig. 8(b).

Figure 9 displays the phase space densities $f_{1,2}\left(x, p_{x}\right)$ and $f\left(x, p_{z}\right)$ at the times $t=50$ and $t=120$. Figure 9(a) demonstrates that the electrons of both beams have been rearranged by the FI. The filaments have not yet reached the stable symmetric configuration, because the most pronounced density minima at $x \approx 1.5$ for beam 2 and at $x \approx 2.5$ for beam 1 are not shifted by $L_{2} / 2$. This asymmetry results in the $E_{B}\left(3 k_{2}, t\right) \neq 0$ and in the broadband $E_{x}(k, t)$ at this time in Fig. 7 . The spatial gradients of $B_{y}(x, t)$ and $E_{x}(x, t)$ are high at $t \approx 50$ and the Lorentz force changes rapidly with $x$, explaining the complex phase space structuring in Fig. 9(b,c). The phase shift of $L_{2} / 2$ of the density maxima of both beams has been reached at $t=120$ in Fig. 9(d). The $B_{y}(x, t)$ is stationary in its rest frame at this time in Fig. 6(a). The electrons are heated up from an initial thermal spread of $p_{x} / p_{0} \approx 0.05$ with $p_{0}=m_{e} v_{b} \Gamma\left(v_{b}\right)$ to a peak value of 
$p_{x} \approx p_{0}$ in Fig. $9(\mathrm{e}, \mathrm{f})$. The mean momentum of each beam varies along $p_{z}$ in response to a drift imposed by $E_{x}(x, t)$ and $B_{y}(x, t)$ but no heating is observed in this direction.

Movie 2 shows the 10-logarithmic phase space density projections $f_{1}\left(x, p_{x}, t\right)$ and $f_{1}\left(x, p_{z}, t\right)$ of beam 1 in the simulation 2 . It demonstrates that only the core electrons in Fig. 9remain spatially confined. The heated electrons, which have in some cases reached a momentum $p_{x}$ that is comparable to the initial beam momentum, are untrapped. The heated electrons move practically freely and they ensure that the beam confinement is not perfect. The trapped electrons maintain the $J_{z}(x, t) \neq 0$ and, thus, the $B_{y}(x, t) \neq 0$. The trapped electrons slowly move to larger values of $x$. The associated shift of $J_{z}(x, t)$ causes the slow drift of $B_{y}(x, t)$ in Fig. 6(a). The movie visualizes the formation of the phase space beams and their evolution into phase space holes in $f_{1}\left(x, p_{x}\right)$.

\section{Discussion}

We have examined here the electron beam filamentation instability (FI) in one dimension and in an initially unmagnetized plasma with immobile ions. The FI has been driven by nonrelativistic symmetric electron beams with the same initial conditions as those considered previously [21, 22]. The electric field along the one-dimensional box, which is oriented orthogonally to the beam velocity vector, can only be generated nonlinearly if the beams are symmetric [20]. The fluid equations show that the relevant nonlinear mechanisms can be the magnetic pressure gradient force (MPGF), the thermal pressure gradient force and a term due to the displacement current. The magnetic tension may become important in multi-dimensional simulations, but not for initial conditions similar to ours [26]. The term due to the displacement current is weak in our simulations.

It has been observed in Ref. [22] that the electrostatic field performs after the saturation of the FI undamped oscillations around a time-stationary background electric field. The amplitude of the oscillatory and of the background electric field are both given by $E_{B}(x, t) \approx-B_{y} d_{x} B_{y}$. The phases of both fields are fixed such, that $E_{x}\left(x, t_{0}\right)=2 E_{B}\left(x, t_{0}\right)$ at the saturation time $t_{0}$. This amplitude ensures that the nonlinear terms due to the MPGF and due to the electrostatic field cancel each other approximately in the fluid equations when the FI saturates. The thermal pressure gradient force did not visibly contribute in the simulation of the small filament pair [26], possibly because of the only modest heating of the initially cool beams. Here we have assessed the importance of the filament size with the help of two 1D PIC simulations, which used two different box lengths that were larger than that of the 1D box in Ref. [22, 26]. The initial conditions for the plasma were otherwise identical.

We summarize our findings as follows. We have demonstrated for both simulations, that $E_{x}\left(x, t \leq t_{0}\right) \approx 2 E_{B}\left(x, t \leq t_{0}\right)$ during the full exponential growth phase and not just at the saturation time $t_{0}$. The FI thus adjusts the electrostatic field during its exponential growth phase such, that the dominant nonlinear terms cancel each other. Magnetic trapping states that the FI saturates, when the magnetic bouncing frequency is comparable to the linear growth rate. The exponential growth rates for the two 
simulations considered here and that in Ref. [22] are close. The amplitude reached by the magnetic field prior to its satuation thus increases with the box length. We found that the electrostatic potential driven by the MPGF is 5-6 times stronger for the box sizes used here than for the short box in Ref. [22], while the initial mean kinetic energy of the electrons is the same. Consequently, the electron heating is stronger and the plasma processes more violent for large filaments. Magnetic trapping is, however, not the exclusive saturation mechanism. The electrostatic forces are comparable in strength to the magnetic forces when the FI saturates [13, 14].

The electrostatic field during the intermittent phase has differed in our two simulations from that observed in Ref. [22]. The movies demonstrated that this phase involves the formation of large nonlinear structures (phase space holes) in the electron distribution, which can result in steep gradients of the thermal pressure and in the generation of solitary (bipolar) electrostatic wave structures that are independent of the fields produced by the FI. The thermal pressure gradient force is comparable to that of the other nonlinear terms, but only over limited spatial intervals. The electric field component along the beam velocity vector has undergone a mode conversion. Its energy leaked into the high-frequency electromagnetic modes [14].

The wavenumber spectrum of the electrostatic field correlated well with that of the MPGF in simulation 1, but the peak electric field overshot the expected one. The electrostatic field performed damped oscillations around $E_{B}$ and both converged eventually to the same value. The wavenumber spectrum of the electrostatic field in simulation 2 deviated from that of the MPGF in the intermittent phase. Its wavenumber spectrum was broadband, while that of the MPGF was quasi-monochromatic. The amplitude modulus of the electrostatic field at the wavenumber, which corresponds to the dominant Fourier component of the MPGF, jumped to the value expected from the MPGF. It did not overshoot and it was non-oscillatory.

Both simulations here have evidenced that the magnetic field driven by the FI organized itself such, that we obtained one oscillation in the simulation box after the intermittent phase. This is remarkable, because the exponential growth rate of the fundamental wavenumber is below that of its first harmonic. Long waves excert a lower MPGF for a given amplitude and the dominance of the fundamental wavenumber may thus result from the lower nonlinear damping of this mode compared to that of its harmonics. The mode with the fundamental wavenumber considered in Ref. [22] has a higher growth rate than its harmonics and the absent mode competition may have facilitated the undamped oscillations around the equilibrium. However, the amplitude of the electrostatic field in the two simulations discussed here eventually converged to that expected from the MPGF and $E_{B}$ is thus a robust estimate for the electrostatic field driven by the MPGF for the considered case. This robustness explains, why a connection between the electrostatic field and the MPGF has been observed in a 2D PIC simulation [26], where no equilibrium can be reached due to the filament mergers.

This estimate does, however, not apply if positrons are present. Their current reduces that of the electrons. If equal amounts of electrons and positrons are present, 
the electrostatic field driven by the MPGF is suppressed alltogether [27]. Mobile protons will react in particular to the stationary electric field [14] and they will modify through their charge modulation the balance between the electrostatic field and the MPGF. Highly relativistic beam velocities will probably also modify the balance between the MPGF and the electron currents it drives. We leave relativistic beams to future work.

Acknowledgements The authors acknowledge the support by Vetenskapsrådet and by the projects FTN 2006-05389 of the Spanish Ministerio de Educacion y Ciencia and PAI08-0182-3162 of the Consejeria de Educacion y Ciencia de la Junta de Comunidades de Castilla-La Mancha. The HPC2N has provided the computer time.

\section{References}

[1] Yang T Y B, Gallant Y, Arons J and Langdon A B 1993 Phys. fluids B 53369

[2] Petri J and Kirk J G 2007 Plasma Phys. Controll. Fusion 49297

[3] Karlicky M, Nickeler D H and Barta M 2008 Astron. Astrophys. 486325

[4] Medvedev MV and Loeb A 1999 Astrophys. J. 526697

[5] Lazar M, Schlickeiser R and Shukla P K 2006 Phys. Plasmas 13102107

[6] Tabak M et al. 1994 Phys. Plasmas 11626

[7] Ruhl H, Sentoku Y, Mima K, Tanaka K A and Kodama R 1999 Phys. Rev. Lett. 82743

[8] Key M H et al. 2008 Phys. Plasmas 15022701

[9] Davidson R C, Wagner C E, Hammer D A and Haber I 1972 Phys. Fluids 15317

[10] Lee R and Lampe M 1973 Phys. Rev. Lett. 311390

[11] Molvig K 1975 Phys. Rev. Lett. 351504

[12] Honda M, Meyer-ter-Vehn J and Pukhov A 2000 Phys. Rev. Lett. 852128

[13] Honda M, Meyer-ter-Vehn J and Pukhov A 2000 Phys. Plasmas 71302

[14] Califano F, Cecchi T and Chiuderi C 2002 Phys. Plasmas 9451

[15] Sakai J I, Schlickeiser R and Shukla P K 2004 Phys. Lett. A 330384

[16] Medvedev M V, Fiore M, Fonseca R A, Silva L O and Mori W B 2005 Astrophys. J. 618 L75

[17] Stockem A, Dieckmann M E and Schlickeiser R 2008 Astrophys. J. 50025002

[18] Bret A, Gremillet L and Bellido J C 2007 Phys. Plasmas 14032103

[19] Bret A, Gremillet L, Benisti D and Lefebvre E 2008 Phys. Rev. Lett. 100205008

[20] Tzoufras M, Ren C, Tsung F S, Tonge J W, Mori W B, Fiore M, Fonseca R A and Silva L O 2006 Phys. Rev. Lett. 96150002

[21] Rowlands G, Dieckmann M E and Shukla P K 2007 New J. Phys. 9247

[22] Dieckmann M E, Kourakis I, Borghesi M and Rowlands G 2009 Phys. Plasmas 16074502

[23] Dawson J M 1983 Rev. Mod. Phys. 55403

[24] Eastwood J W 1991 Comput. Phys. Commun. 64252

[25] Sharma R P, Tripathi Y K and Kumar A 1987 Phys. Rev. A 353567

[26] Dieckmann M E 2009 Plasma Phys. Controll. Fusion in press (2009)

[27] Dieckmann M E, Shukla P K and Stenflo L 2009 Plasma Phys. Controll. Fusion 51065015 